\title{
ANÁLISIS DE LAS PRINCIPALES TENDENCIAS DE INNOVACIÓN EN NANOTECNOLOGÍAS DE ALIMENTOS: UNA APROXIMACIÓN A SU ESTUDIO A PARTIR DE VIGILANCIA TECNOLÓGICA
}

\author{
Analysis of main trends in food nanotechnology innovation: a technology surveillance-based \\ approach
}

Jhonjali García-Mosquera

Administrador de Empresas. Universidad Tecnológica del Chocó, Colombia, jhojalisk2@gmail.com

\section{Eliana María Villa-Enciso iD}

PhD. (c) en Ingeniería Industria y Organizaciones, Universidad Nacional de Colombia, Colombia, emvilla@unal.edu.co

\section{Cómo referenciar / How to cite}

García-Mosquera, J., \& Villa-Enciso, E. M. (2018). Análisis de las principales tendencias de innovación en nanotecnologías de alimentos: una aproximación a su estudio a partir de vigilancia tecnológica. Revista CEA, 4(8), 95-115. https://doi.org/10.22430/24223182.1118

Recibido: 01 de marzo de 2018

Aceptado: 13 de junio 2018

Resumen: en el estudio se analizan las principales tendencias en producción científica y tecnológica en el área de nanotecnología de alimentos. El propósito se centró en conocer su evolución y así poder identificar oportunidades potenciales de innovación, con base en sus principales usos y aplicación en la industria alimenticia. Se utilizó la Vigilancia Tecnológica, indicadores cienciométricos, así como la teoría de redes y grafos para la visualización y análisis del comportamiento de las principales áreas de investigación y desarrollo tecnológico, con base en el tema de estudio. Se identificaron ocho potenciales oportunidades de innovación en el área: nanopartículas, nanomateriales, nanocompuestos, nanoemulsión, nanoencapsulación, microencapsulación, nanorecubrimientos y nanosensores; las cuales están influyendo notablemente en el desarrollo de la industria de alimentos y se han convertido en elementos claves de investigación y desarrollo tecnológico a nivel mundial. El uso y aplicación de la nanotecnología en la industria de alimentos han aumentado rápidamente en las últimas décadas y su constante evolución ha tenido una gran contribución en el desarrollo de la industria.

Palabras clave: nanotecnología, innovación, alimentos, producción científica, desarrollo tecnológico.

\begin{abstract}
This study analyzes the main trends in scientific and technological production in the field of food nanotechnology. The purpose is to describe their evolution and thus identify potential opportunities for innovation based on their main uses and applications in the food industry. Technology Surveillance, scientometric indicators, and graph and network theory were used to visualize and analyze the behavior of the main research fields and technological development of the subject under study. We identified 8 potential innovation opportunities in the area: nanoparticles, nanomaterials, nanocomposites, nanoemulsions, nanoencapsulation, microencapsulation, nanorecovering, and nanosensors. All of them are significantly influencing the development of the food industry and have become key
\end{abstract}


elements of research and technological progress worldwide. The uses and applications of nanotechnology in the food industry have rapidly expanded in recent decades, and their constant evolution has made a great contribution to the development of this sector.

Keywords: nanotechnology, innovation, food, scientific production, technological development.

\section{INTRODUCCIÓN}

Con la creciente demanda por parte de los consumidores de nuevos productos, con características duraderas, seguras y con propiedades nutricionales saludables, la industria alimentaria lleva años investigando en el desarrollo de nuevas tecnologías que puedan responder a esta tendencia (Fundación Vasca para la Seguridad Alimentaria, 2012; Kalpana, Anshul, \& Rao, 2013; Steenis \& Fischer, 2016; Robles, García et al., 2016; Granqvist \& Ritvala, 2016; Bowman \& Ludlow, 2017), planteando la nanotecnología como una importante herramienta que ha revolucionado a la industria alimentaria y que su aplicabilidad ha abarcado desde el desarrollo de materiales a una escala de nanómetros con el fin de detectar contaminantes, controlar la trazabilidad y sistema de envasado hasta avanzar en el desarrollo molecular, funcional y microbiológico de los alimentos (Erdem, 2015; He \& Hwang, 2016; Eduok \& Coulon, 2017; Singh et al., 2017; Eleftheriadou \& Demokritou, 2017; Thiruvengadam, \& Chung, 2018).

El siguiente estudio de Vigilancia Tecnológica, en adelante VT, se basó en explorar y examinar las principales tendencias en producción científica y tecnológica a nivel mundial sobre nanotecnología, como herramienta fundamental en la industria de alimentos, esto con el fin de conocer su evolución y así poder identificar oportunidades potenciales de innovación con base a sus usos $\mathrm{y} / \mathrm{o}$ aplicabilidad. Por ello, fue necesario apoyarse de indicadores cienciométricos (Macías, 2001; Elango, Rajendran, \& Bornmann, 2015), así como la teoría de redes y grafos (Molina, 2004; Vélez, 2010; Wasserman \& Faust, 2011; Robledo, Osorio, \& López, 2014; Pinto \& González, 2014; Yandy, 2015) como mecanismos metodológicos complementarios para visualizar y analizar el comportamiento del tema en análisis. En este orden, el documento se estructuró de la siguiente manera: en la primera sección, se hace una breve revisión de literatura sobre el tema en estudio; en la segunda sección, se diseña y define una ecuación de búsqueda a partir de términos clave identificados en la sección anterior, a su vez se describe detalladamente la metodología abordada; en la tercera sección, se presentan los principales hallazgos 0 resultados del estudio según la metodología; y finalmente, en la sección cuatro, se realizan las discusiones y conclusiones pertinentes.

\section{MARCO TEÓRICO}

La nanotecnología es un área nueva de investigación que empezó a tomar fuerza en la década de los 80 y 90, gracias a los trabajos de Drexler, Feynman, Rorher y Binnig, por mencionar algunos (Serena, 2002). Su desarrollo y surgimiento se alude a la necesidad que ha tenido el ser humano de elaborar materiales industriales, con base en la creación de nuevas clases de estructuras moleculares originales (Molins, 2008; Steenis \& Fischer, 2016; Otles S., Yalcın B., 2015; Aouada, de Moura, 2015; Viberg, 2017), para hacer frente a los principales problemas de salud, energía y medio ambiente que aquejan a la población mundial (Masami et al., 2013; Tomiko, 2013; Sunil, K., Paresh \& Kulkarni, 2013; Sodano, Gorgitano, Quaglietta, \& Verneau, 2016; Cundy, 2017; Bautista et al., 2017; Sougata, Gandhi \& Jana, 2017).

Este concepto abarca diversas áreas de aplicación en el sector industrial, como nanomateriales y nanosensores, la producción 
de nanopartículas y materiales nanoestructurados (Gomes, Pastore, Martins, \& Francisco, 2015; Lacey, 2017; Busquets, 2017), sin embargo, en la industria alimentaria, esta tecnología se ha constituido en una herramienta de investigación y desarrollo tecnológico fundamental, gracias a las exigencias de los consumidores en adquirir alimentos más funcionales, duraderos y nutritivos (Kalpana, Anshul, \& Rao, 2013; Singh, 2016; Fornari, Grandi, Menegatti, \& Hofacker, 2016; Dilmaçünal, 2017; Pathak, 2017). A continuación, se hace una breve descripción teórica de esta nueva herramienta y sus principales aportes en la industria de alimentos.

En primer lugar, la nanotecnología es catalogada como una nueva revolución tecnológica que ayudará en las próximas década a solucionar los más diversos problemas; a partir de sus estudios se pueden encontrar nuevas formas de combatir el cáncer, diseñar medios eficientes para potabilizar agua, construir sistemas de almacenamiento de energía más pequeños y de mayor duración, envases que conservan mejor los alimentos entre otras promesas de esta nueva área tecnológica (Foladori \& Invernizzi, 2012; Amini, Seyed; Gilaki, Marzieh; Karchani, 2014; Gomes, Pastore, Martins, \& Francisc, 2015; Sodano, Gorgitano, Quaglietta, \& Verneau, 2016; Busquets \& Mbundi, 2017).

Según Gomes, Pastore, Martins, \& Francisc (2015); Sodano et al., (2016) esta hace referencia a un conjunto de tecnologías, técnicas y procedimientos utilizadas para construir un nuevo material a escala nanométrica y es considerada una tendencia actual en la ciencia y la tecnología del siglo presente.

Por otro lado, entre las áreas que pueden beneficiarse de esta nueva tecnología, la industria alimentaria es, de hecho, uno de los sectores que atrae más inversiones (Gomes, Pastore, Martins, \& Francisc, 2015); e incluye el desarrollo de nuevos materiales funcionales, productos, envases y métodos para mejorar la inocuidad de los alimentos (Echegoyen, 2015; Sadeghi, Rodríguez, Yao, \& Kokini, 2017; Jurewicz, 2017).

\section{METOdOLOGÍA}

El estudio se llevó a cabo mediante una vigilancia tecnológica apoyada en indicadores cienciométricos (Barbosa, 2012), así como de la teoría de redes y grafos que permitiera explorar, analizar y visualizar el comportamiento del área en estudio, con el fin de identificar oportunidades de innovación potenciales con base en sus principales usos y/o aplicabilidad. Esta metodología se basó en 3 fases:

F1. Planeación de VT. Aquí se define el objetivo y alcance del estudio, así como la definición de bases de datos para la recuperación de la información de carácter estructurada.

F2. Estrategia para la búsqueda y análisis de la información. En esta fase, se definen los términos de búsqueda con base a elementos claves identificados en la revisión de literatura y se diseña una ecuación, la cual fue usada para la recuperación, selección y construcción de indicadores y grafos.

F3. Tratamiento y análisis de la información. Finalmente, en esta fase se analizan: a) Tendencias actuales en producción científica teniendo en cuenta: número de publicaciones científicas durante el año, principales países con publicaciones científicas, red de colaboración de los países líderes en publicaciones científicas, revistas líderes, red de colaboración de revistas, principales autores en el área, centralidad de grado e intermediación de los autores líderes. b) Tendencias en producción tecnológica, se analiza: número de patentes publicadas a través de los años, principales países e instituciones con desarrollo tecnológico y se 
finaliza con la red de las principales tecnologías identificadas, las cuales fueron consideradas como parte de las oportunidades de innovación potencial en el área de nanotecnología de alimentos.
En la figura 1 se puede observar esquemáticamente la metodología utilizada:

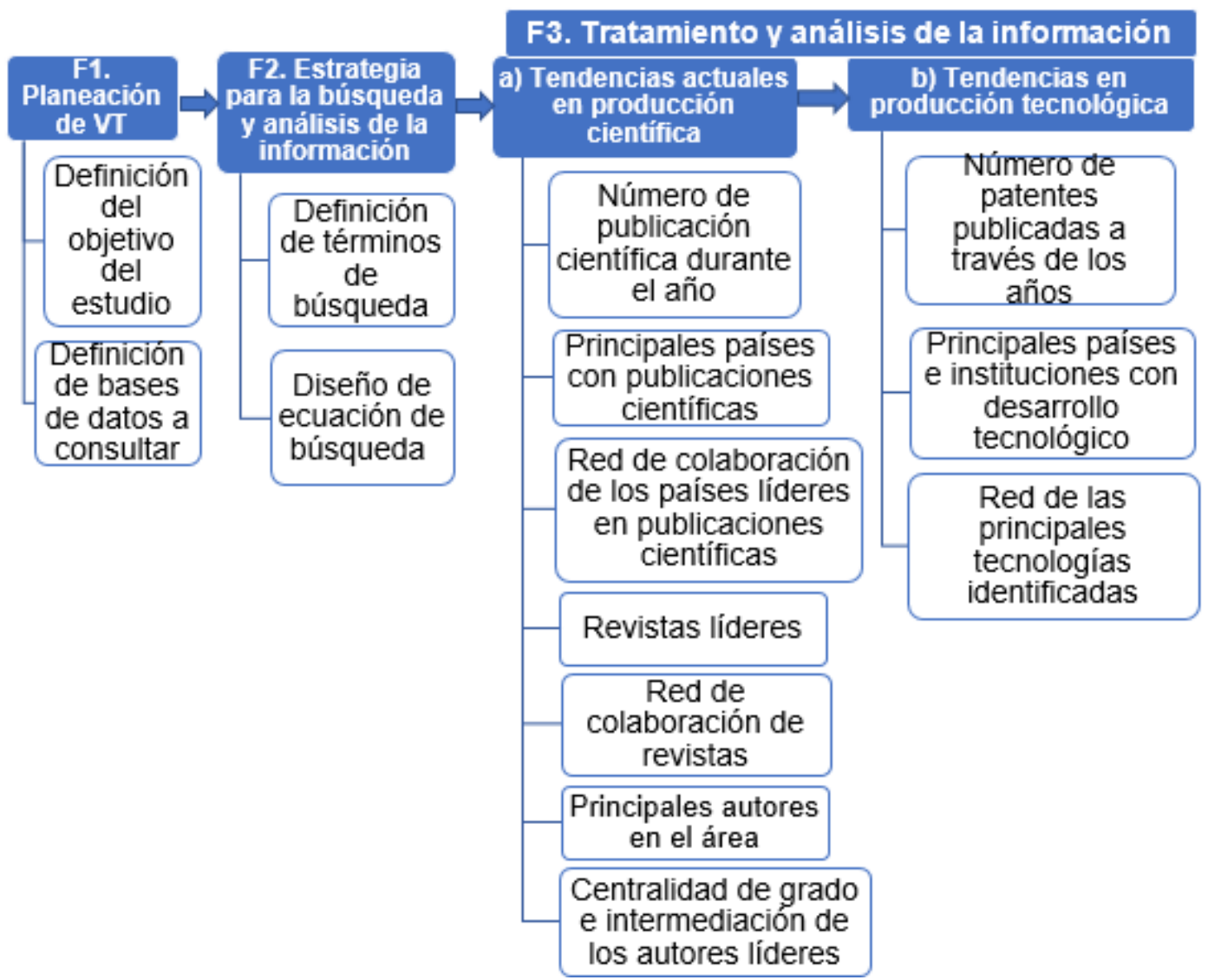

Figura 1. Metodología de VT aplicada en el estudio

Figure 1. TS method applied in the study

Fuente: elaboración propia.

\section{RESULTADOS}

En este apartado, se presentan los principales hallazgos del estudio teniendo en cuenta la metodología descrita con anterioridad. En este sentido se realizó lo siguiente:

\section{F1. Planeación de VT}

Con el desarrollo de este estudio de VT aplicado en la industria de los alimentos, se planteó conocer: ¿Cuáles son las principales tendencias en producción científica y tecnológica en el campo de nanotecnología en alimentos a nivel mundial que permitan la identificación de potenciales oportunidades de innovación? Posteriormente, se definen las bases de datos a consultar como estrategia de recolección de información e insumo clave para alimentar la revisión de literatura y diseñar la ecuación de búsqueda. Estas bases de datos se describen en la

Tabla 1.

\section{F2. Estrategia para la búsqueda y análisis de la información}

Para dar respuestas al objetivo planteado en el estudio, fue necesario definir los términos de búsqueda, producto de los resultados de la 
revisión de literatura, y diseñar dos ecuaciones de búsqueda necesarias para la recolección de literatura científica estructurada, así como la recuperación de la producción tecnológica.
Dicha información fue procesada para la construcción de los indicadores cienciométricos y grafos de análisis. Este proceso se muestra en la Tabla 2.

Tabla 1. Descripción de las bases de datos definidas en el estudio Table 1. Description of the databases used in the study

\begin{tabular}{|c|c|l|}
\hline Tipo & Bases de datos & \multicolumn{1}{c|}{ Descripción } \\
\hline \multirow{2}{*}{$\begin{array}{c}\text { Producción } \\
\text { científica }\end{array}$} & Scopus $^{\circ}$ & $\begin{array}{l}\text { Es la mayor base de datos de resúmenes hasta ahora vista en el } \\
\text { mundo, con 20.500 publicaciones (85\% de las cuales están indizadas } \\
\text { con vocabulario controlado) procedentes de más de 5.000 editoriales } \\
\text { internacionales. }\end{array}$ \\
\cline { 2 - 4 } & $\begin{array}{c}\text { The SCImago } \\
\text { Journal \& Country } \\
\text { Rank }\end{array}$ & $\begin{array}{l}\text { Es un portal público que incluye las revistas y los indicadores } \\
\text { científicos de los países desarrollados a partir de la información } \\
\text { contenida en la base de datos Scopus }{ }^{\circledR} \text { (Elsevier BV). }\end{array}$ \\
\hline $\begin{array}{c}\text { Producción } \\
\text { tecnológica }\end{array}$ & Patentscope & $\begin{array}{l}\text { Proporciona acceso a las solicitudes internacionales del tratado de } \\
\text { cooperación en materia de patentes en formato de texto completo, } \\
\text { el día de la publicación, y los documentos de patentes de las oficinas } \\
\text { nacionales y regionales de patentes participantes. }\end{array}$ \\
\hline
\end{tabular}

Fuente: elaboración propia con base a Elsevier (2015); Rodríguez (2015); WIPO (2017).

Tabla 2. Definición de términos claves para el diseño de ecuación de búsqueda

Table 2. Selection of keywords to design the search equation

¿Cuáles son las principales tendencias en producción científica y tecnológica en el campo de nanotecnología en alimentos a nivel mundial que permitan la identificación de y desarrollo de potenciales oportunidades de innovación?

\begin{tabular}{|l|l|l|}
\hline Palabra clave & \multicolumn{2}{|c|}{ Sinónimos } \\
\hline Nanotechnology in food & Nanofood & Functional Nanofoods \\
\hline
\end{tabular}

Fuente: elaboración propia con base a la revisión de literatura.

\section{Diseño de ecuaciones de búsqueda}

Como se mencionó con anterioridad, a partir de estos términos claves se definieron las siguientes ecuaciones de búsqueda:

$\checkmark$ Producción científica: TITLE-ABS-KEY ((“Nanotechnology in food"*) OR (“Nanofood") OR ("Functional Nanofoods")) AND PUBYEAR > 2007 AND PUBYEAR < 2018

$\checkmark$ Producción tecnológica: ((Nanotechnology in food) OR (Nanofood) OR (Functional Nanofoods)) AND DP: [2008 to 2017]

\section{F3. Tratamiento y análisis de la información}

De acuerdo con el diseño de las ecuaciones, se procedió a la búsqueda de la información con respecto a la producción científica y tecnológica. Para la producción científica se consultó la base de datos Scopus ${ }^{\circ}$, de la cual se recuperaron 153 publicaciones en un periodo comprendido entre el 2008 hasta el 2017. Por otro lado, con respecto a la producción tecnológica, se consultó la base de datos de la WIPO - Patenstcope de la que se pudo obtener 4.342 invenciones entre el 2008 al 2017. 
4.1 Tendencias actuales de la producción científica en nanotecnologías de alimentos

De acuerdo con la información obtenida de la base de datos Scopus ${ }^{\circ}$, se ha registrado un total de 153 publicaciones en el área de estudio en un periodo comprendido entre 2008 y 2017. Estas publicaciones han experimentado constantes fluctuaciones en lo corrido de la última década. Sin embargo, en los últimos 5 años, la tendencia marca que en promedio las publicaciones se han mantenido por encima de los 14 documentos. En el Gráfico 1 se puede apreciar dicho comportamiento y las fluctuaciones, notándose el mayor crecimiento de producción científica entre los años 2013 y 2014.

\section{Principales países con publicaciones científicas}

Entre los principales países con participación en la producción científica, con respecto al tema de interés, se destacan: Estados Unidos e India como líderes, con 23 publicaciones, relacionadas con la importancia que tiene la nanotecnología para la conservación y transformación de alimentos; seguidos del Reino Unido, con 19; Bélgica 12; y finalmente, Corea del Sur con 10. En el Gráfico 2 se describe el top diez de estos países.

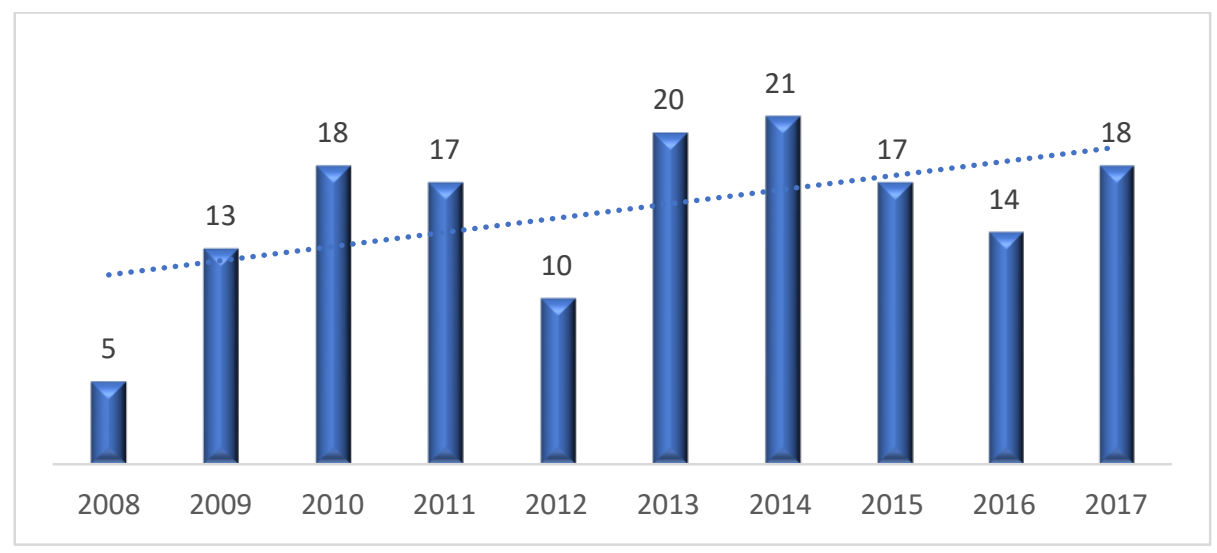

Gráfico 1. Número de publicaciones a través de los años

Graph 1. Number of publications over the years

Fuente: elaboración propia base a Scopus ${ }^{\circ}$

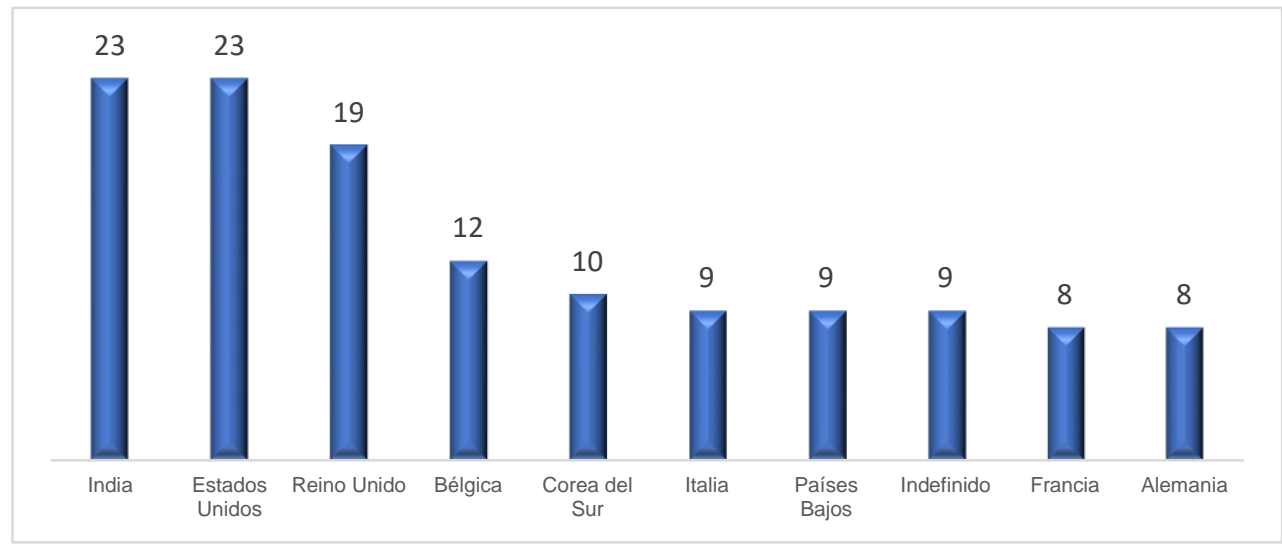

Gráfico 2. Principales países con publicaciones científicas

Graph 2. Top countries in scientific publications

Fuente: elaboración propia con base a Scopus ${ }^{\circ}$ 
Red de colaboración de los países líderes en publicaciones científicas

Sumado a lo anterior, se construyó una red que muestra la colaboración de los países líderes en producción científica. Esta red está conformada por nodos y aristas, los cuales representan los países y las relaciones e interacciones generadas, a medida que se realizan trabajos o se crean vínculos de acuerdo con la publicación de temas comunes. Con base en esto, en la Figura 2 se visualizan las diversas interacciones producidas, hasta la fecha, entre los principales países que colaboran en la generación y transferencia de conocimiento en el área de nanotecnología de alimentos. Los colores de los nodos y aristas indican relaciones directas $y$, entre más grande sean, significa mayor visibilidad, peso y grado de colaboración en la red, siendo entonces Estados Unidos, India y Reino Unido los países con mayor influencia y participación en la red.

\section{Revistas líderes en publicación}

Según la información obtenida de la base de datos de Scopus ${ }^{\circ}$, entre las revistas líderes en producción científica se destaca: Rsc Nanoscience and Nanotechnology, con un total de 5 documentos publicados en el 2017, relacionados con ingredientes genéticamente modificados, la nanotecnología en envases y su uso en la ciencia de los alimentos. Otras de las revistas líderes se muestran en el Gráfico 3.

De acuerdo con la base de datos Scimago Journal \& Country Rank, que mide el nivel de prestigio de las revistas más importantes del mundo en la comunidad científica (Rodríguez, 2015), de las principales revistas mencionadas con anterioridad, Trends In Food Science and Technology, de Netherlands presenta el mayor número de factor de impacto (142); en el periodo comprendido entre 1997 y 2015 se ha ubicado entre segundo y primer quartíl (Q1Q2) en las áreas de ciencias agrícolas y biológicas, ciencia de los alimentos y biotecnología. A continuación, se encuentra la revista japonesa "Biological and Pharmaceutical Bulletin", con el segundo mayor factor de impacto (100) y manteniendo su nivel de prestigio entre el primer y segundo quartil (Q1-Q2) en las áreas de farmacología, toxicología y medicina. En la Tabla 3 se describe dicha información con base en las 5 revistas identificadas en el estudio.

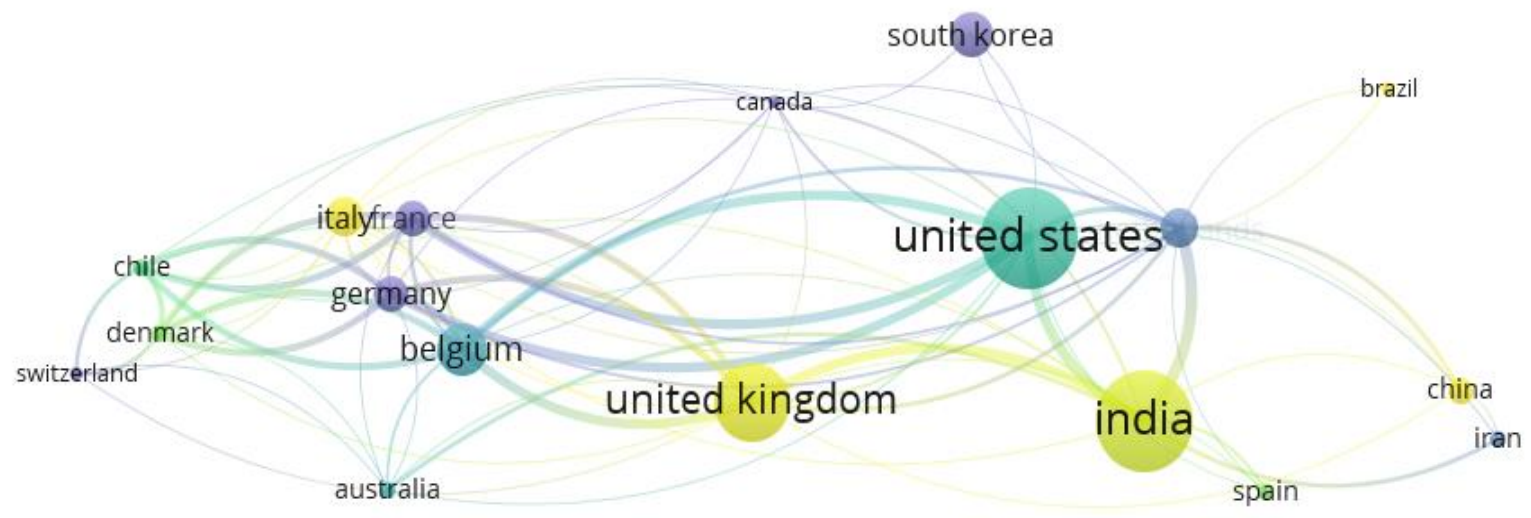

Figura 2. Red de colaboración de los países líderes en publicaciones científicas

Figure 2. Collaboration network of leading countries in scientific publications Fuente: elaboración propia con base a VOSviwer ${ }^{\oplus}$ 


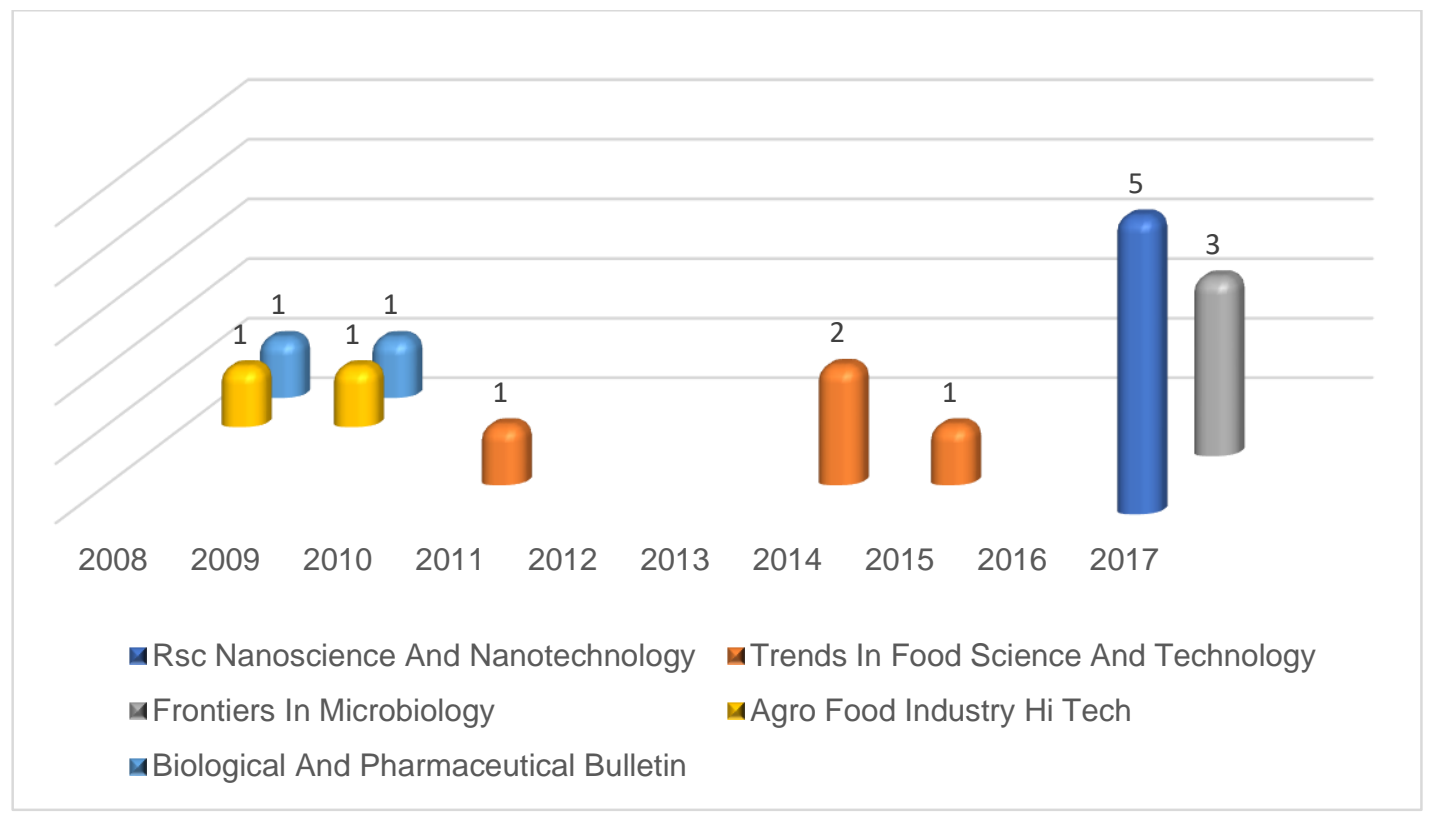

Gráfico 3. Revistas líderes en publicación

Graph 3. Leading journals in publications

Fuente: elaboración propia con base a Scopus ${ }^{\circ}$

Tabla 3. Descripción e historial cienciométrico de la revista líderes en el área

Table 3. Description and scientometric history of leading journals in the field

\begin{tabular}{l|c|c|c|c}
\hline \multicolumn{1}{c|}{ Source } & Country & Subject Area and Category & \multicolumn{2}{c}{ Historial cienciométrico } \\
\hline $\begin{array}{l}\text { Trends in Food Science } \\
\text { and Technology }\end{array}$ & Netherlands & $\begin{array}{c}\text { ciencias agrícolas y biológicas, } \\
\text { ciencia de los alimentos y } \\
\text { biotecnología. }\end{array}$ & H-Index & $\begin{array}{c}\text { Quartiles } \\
\text { (1997-2015) }\end{array}$ \\
\cline { 4 - 5 } $\begin{array}{l}\text { Biological and } \\
\text { Pharmaceutical Bulletin }\end{array}$ & Japan & $\begin{array}{c}\text { pharmacology, toxicology and } \\
\text { medecine }\end{array}$ & 100 & Q2-Q1 \\
\hline $\begin{array}{l}\text { Frontiers in } \\
\text { Microbiology }\end{array}$ & Switzerland & $\begin{array}{c}\text { immunology and microbiology } \\
\text { microbiology, medicine }\end{array}$ & 69 & Q3-Q1 \\
\hline $\begin{array}{l}\text { RSC Nanoscience and } \\
\text { Nanotechnology }\end{array}$ & $\begin{array}{c}\text { United } \\
\text { Kingdom }\end{array}$ & $\begin{array}{c}\text { chemical engineering } \\
\text { bioengineering; } \\
\text { materials science }\end{array}$ & 6 & Q4-Q3 \\
\hline $\begin{array}{l}\text { Agro Food Industry Hi- } \\
\text { Tech }\end{array}$ & Italy & $\begin{array}{c}\text { agricultural and biological } \\
\text { sciences } \\
\text { food science }\end{array}$ & 16 & Q4-Q3 \\
\hline
\end{tabular}

Fuente: elaboración propia.

Red de colaboración de revistas líderes en nanotecnología en alimentos

En la Figura 3, se ilustra la red de colaboración de revistas líderes en la generación y transferencia de conocimiento en el área de interés. Según este grafo, las revistas con mayor participación y peso en la red actúan como ejes centrales, el tamaño de sus nodos es más sobresalientes y las relaciones e interacciones directas están definidas por los tamaños de las aristas.

En este caso, Trends in Food Science and Technology figura como la revista científica más importante en la red y actúa como puente central entre las demás revistas con publicaciones relacionadas. 


\section{Principales autores en el área}

Entre los principales autores en el área, se destacan Bieberstein, A.; Blanchemanche, S.; Marette, S.; Roosen, J. y Vandermoere, F., debido a que registran el mayor número de publicaciones en el área de estudio hasta la fecha de análisis con 7 documentos cada uno. En el Gráfico 4 se puede apreciar dicha información.

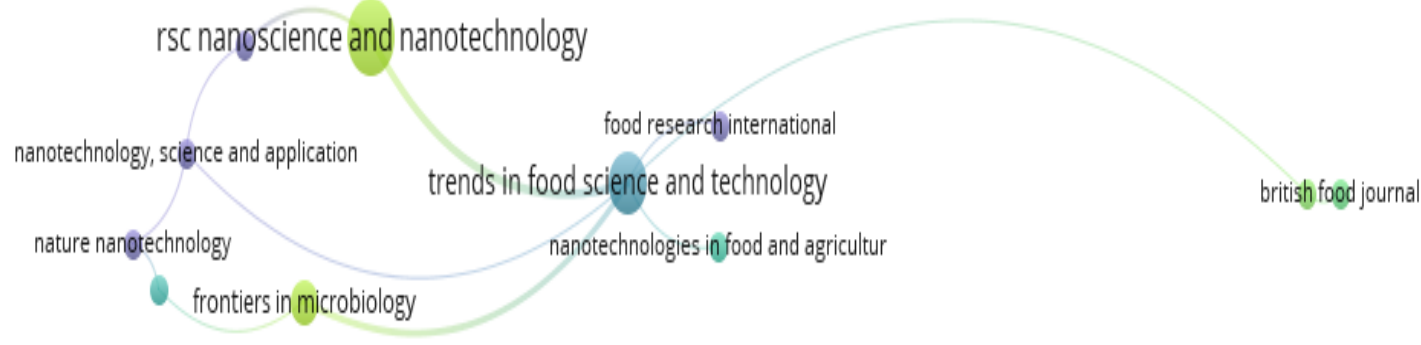

Figura 3. Red colaboración de revistas con publicaciones en el área de estudio

Figure 3. Collaboration network of journals with publications in the field under study Fuente: elaboración propia con base a VOSviwer ${ }^{\circledR}$.

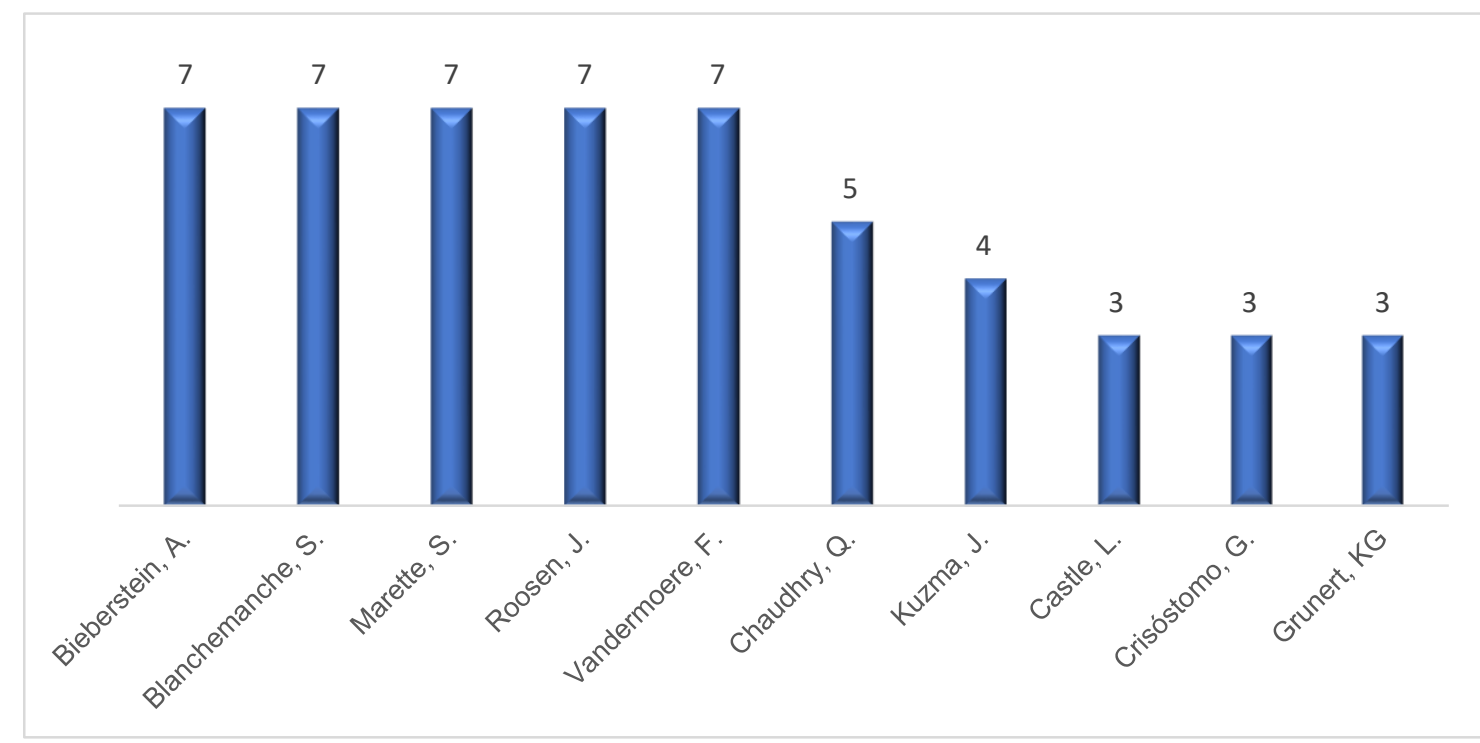

Gráfico 4. Principales autores con publicaciones en el área

Graph 4. Main authors with publications in the field

Fuente: elaboración propia con base en Scopus ${ }^{\circ}$

Haciendo un análisis del perfil de los principales autores con producción científica en el área de estudio, se pudo conocer que los documentos publicados fueron realizados de manera colaborativa bajo la misma afiliación institucional entre los años 2009 y 2013, distribuidas entre artículos (5), capítulos de libros (1), review (1), como se puede apreciar en la Tabla 4. 
Tabla 4. Perfil de los principales autores en el área

Table 4. Profile of the main authors in the field

\begin{tabular}{|c|c|c|c|c|}
\hline Autores & $\begin{array}{c}\text { Filiación } \\
\text { institucional } \\
\text { (\# de } \\
\text { artículos) } \\
\end{array}$ & $\begin{array}{c}\text { Frases de } \\
\text { interés } \\
\text { (frecuencia) }\end{array}$ & $\begin{array}{c}\text { Tendencias de } \\
\text { publicación }\end{array}$ & Tipos de documentos \\
\hline Bieberstein, $\mathrm{A}$. & \multirow{5}{*}{$\begin{array}{l}\text { Technische } \\
\text { Universitat } \\
\text { Munchen (7) }\end{array}$} & \multirow{5}{*}{$\begin{array}{l}\text { Experimental } \\
\text { Economics (3) } \\
\text { Nanotechnolog } \\
\text { y (3) }\end{array}$} & \multirow{5}{*}{ 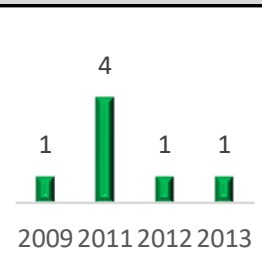 } & \multirow{5}{*}{ - Article "Book Chapter = Review } \\
\hline Blanchemanche, S. & & & & \\
\hline Marette, S. & & & & \\
\hline Roosen, Jutta. & & & & \\
\hline Vandermoere,F. & & & & \\
\hline
\end{tabular}

Fuente: elaboración propia con base en Scopus

En esta línea, en la Tabla 5, se hace una breve descripción de las principales áreas temáticas de interés e historial cienciométrico del top 5 de autores con publicaciones relacionadas. Para ello se tiene en cuenta el total de documentos publicados, número de citaciones realizadas por documentos, así como también las publicaciones desarrolladas en coautorías y el factor de impacto que muestren el nivel de prestigio de cada uno de estos autores, basado en el número de artículos publicados frente al índice de impacto o citaciones recibidas por cada una de estas publicaciones.

Según lo anterior, los autores con mayor número de publicaciones y $h$ - Index, son: Marette, Stéphan con un total de 73 documentos y un factor de impacto de 18; lo que significa que al menos 18 del total de artículos publicados, han recibido al menos 18 citaciones; seguido de Blanchemanche, S., con 23 documentos y un factor de impacto de 13.

Tabla 5. Áreas de interés e historial cienciométrico de los principales autores Table 5. Fields of interest and scientometric history of main authors

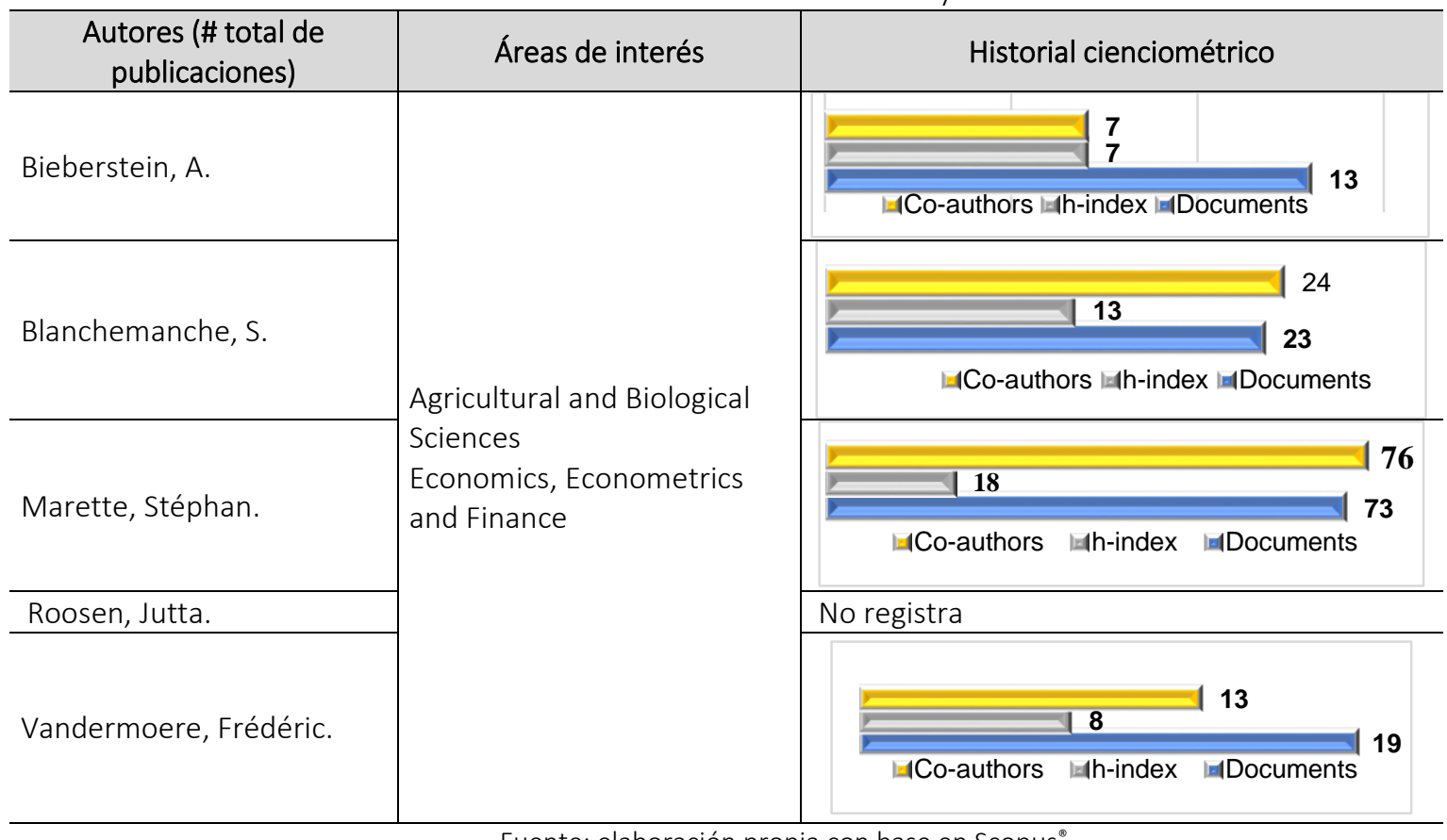

Fuente: elaboración propia con base en Scopus ${ }^{\oplus}$ 


\section{Centralidad de grado e intermediación}

Para el cálculo de estos indicadores, se utilizó el software Gephi', con el cual se elaboraron dos grafos, teniendo en cuenta las siguientes características descritas en la

Tabla 6.

Con base en la Figura 4 y en la tabla 7, se puede apreciar los vínculos que establecen los autores líderes en colaboración para la producción de documentos científicos, de acuerdo al tema en análisis. En este caso, Blanchemanche, S. sigue destacándose como uno de los autores líderes, registrando el mayor grado de colaboración e intermediación en la red, con valores de 14 y 13,4; lo que indica que posee la mayor participación promedio en la construcción y publicación de documentos con respecto de otros autores.

Tabla 6. Parámetros del grafo para el cálculo de la centralidad

Table 6. Parameters of the graph to calculate centrality

\begin{tabular}{|c|c|c|c|}
\hline Tipo de grafo & Descripción de los nodos & Vínculos & Medidas desarrolladas \\
\hline No dirigido & 420 autores & $\begin{array}{l}\text { Artículos } \quad(1146 \\
\text { aristas) }\end{array}$ & $\begin{array}{l}\text { Centralidad de grado } \\
\text { Centralidad de intermediación }\end{array}$ \\
\hline
\end{tabular}

Fuente: elaboración propia con base en software Gephi.

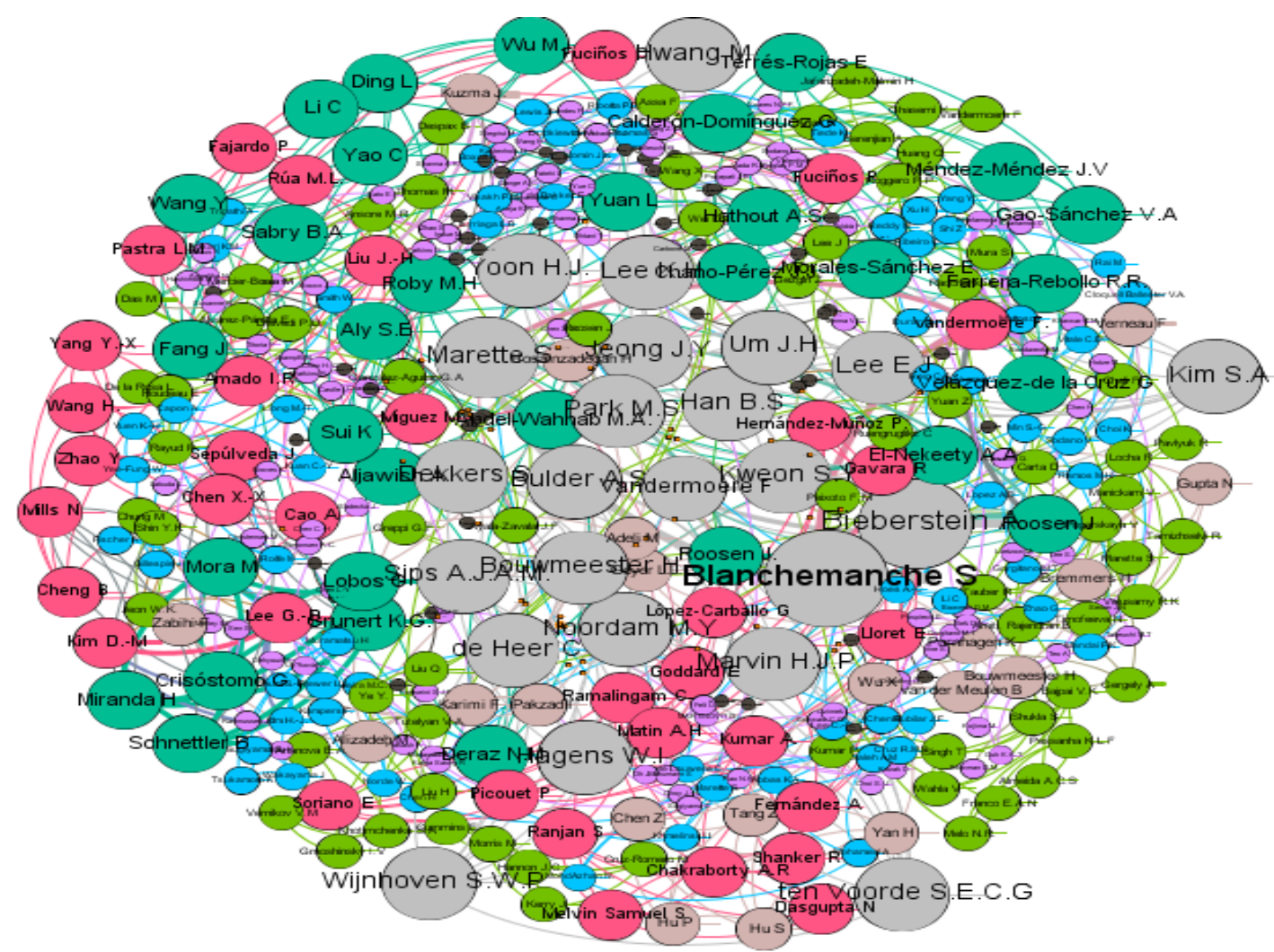

Figura 4. Centralidad de grado e intermediación de los autores líderes

Figure 4. Degree centrality and intermediation of leading authors Fuente: elaboración propia con base a software Gephi". 
Tabla 7. Medidas de centralidad de grado e intermediación de la red

Table 7. Measurements of degree centrality and intermediation of the network

\begin{tabular}{l|l|l}
\hline Autor & Grado de centralidad & $\begin{array}{l}\text { Centralidad de } \\
\text { intermediación }\end{array}$ \\
\hline Blanchemanche S & 14 & 13,4 \\
\hline Bieberstein A & 13 & 8,2 \\
\hline Marette S & 12 & 8 \\
\hline Verneau F & 7 & 6 \\
\hline Kuzma J & 7 & 5,3 \\
\hline \multicolumn{2}{r}{ Fuente: elaboración propia con base a software Gephi }
\end{tabular}

Tendencias actuales en producción tecnológica en el área de nanotecnología en alimentos

Para el análisis de las tendencias en producción científica, se hizo necesario conocer paralelamente el comportamiento del desarrollo tecnológico en el área de estudio. Para tal fin, se consultó la base de datos de WIPO - Patenstcope a través de la ecuación de búsqueda, en un periodo comprendido entre 2008 y 2017, recuperando en total 4.342 invenciones. En la Tabla 8, se hace un resumen de los países y oficinas con publicaciones a acerca de las invenciones desarrolladas en el campo, instituciones y empresas con participación en el desarrollo tecnológico, los principales inventores, así como el comportamiento de las publicaciones a través del tiempo.

Tabla 8. Descripción de las tendencias actuales en producción tecnológica Table 8. Description of current trends in technological production

\begin{tabular}{|c|c|c|c|c|c|c|c|c|c|}
\hline \multicolumn{10}{|c|}{ INFORMACIÓN DE PATENTES EN EL CAMPO DENANOTECHNOLOGY IN FOOD } \\
\hline Ecuación & \multicolumn{9}{|c|}{ (Nanotechnology in food) OR (Nanofood) OR (Functional Nanofoods)) AND DP:[2008 to 2017] } \\
\hline $\begin{array}{c}\mathrm{N}^{0} \mathrm{de} \\
\text { resultados }\end{array}$ & 26.271 & $\begin{array}{c}\text { Fecha de } \\
\text { consulta }\end{array}$ & $\begin{array}{l}\text { Agosto } \\
10 / 2017 \\
\end{array}$ & Base de datos & PatentScope & Periodo de consulta & \multicolumn{3}{|c|}{$2008-2017$} \\
\hline \multicolumn{2}{|c|}{ Países } & \multicolumn{2}{|c|}{ Código IPC } & \multicolumn{2}{|l|}{ Instituciones o Empresas } & \multicolumn{2}{|c|}{ Principales Inventores } & \multicolumn{2}{|c|}{ Publicaciones por año } \\
\hline País u Oficina & $¥$ Patentes & Código & \# Patentes & Empresa & \# Patentes & Nombre & \# Patentes & Año & \# Patentes \\
\hline Estados Unid & 2.205 & $\mathrm{~A} 61 \mathrm{~K}$ & 1736 & Massachusetts institute of technology & 64 & Sung hsing-wen & 55 & 2008 & 262 \\
\hline \begin{tabular}{|l|l} 
PCT \\
\end{tabular} & 1.178 & G01N & 676 & Massachusetts institute of technology & 60 & Tu hosheng & 55 & 2009 & 309 \\
\hline Australia & 293 & $\mathrm{C} 12 \mathrm{~N}$ & 522 & Moderna therapeutics, inc. & 57 & Antonin de fougerolles & 41 & 2010 & 392 \\
\hline Oficina Europ & 288 & $\mathrm{~A} 61 \mathrm{P}$ & 416 & Gp medical, inc. & 52 & Tirtha chakraborty & 35 & 2011 & 397 \\
\hline Canadá & 152 & B82Y & 294 & National tsing hua university & 52 & De fougerolles antonin & 29 & 2012 & 392 \\
\hline \begin{tabular}{|l|} 
Japón \\
\end{tabular} & 60 & $\mathrm{CO} 07 \mathrm{~K}$ & 288 & \multirow{2}{*}{ 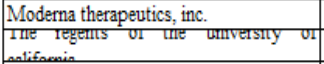 } & 50 & Eugenio minvielle & 27 & 2013 & 445 \\
\hline China & 39 & C12Q & 283 & & 47 & Minvielle eugenio & 25 & 2014 & 536 \\
\hline República de Q & 30 & $\mathrm{~A} 01 \mathrm{~N}$ & 213 & President and fellows of harvard college & 45 & Eugenio M & 22 & 2015 & 494 \\
\hline Federación R & 19 & $\mathrm{CO7D}$ & 187 & President and fellows of harvard college & 42 & Sonaje kiran & 21 & 2016 & 586 \\
\hline Israel & 17 & G06F & 177 & & & Ng pei-sze & 19 & 2017 & 529 \\
\hline & & & & & & & & Total & 4342 \\
\hline
\end{tabular}

Fuente: elaboración propia con base a WIPO - Patenstcope

Número de patentes publicadas a través de los años: Según la información reportada por la base de datos de la WIPO - Patenstcope ${ }^{\circ}$, durante los últimos 10 años se han patentado un total de 4.342 invenciones con respecto al campo de análisis, siendo el año 2016 el de mayor producción tecnológica. En el Gráfico 5, se refleja los niveles de crecimiento y fluctuaciones de estas producciones de un periodo a otro, con una marcada tendencia de 
crecimiento acelerado entre el 2008 y 2014, alcanzado su mayor producción en el 2016 con 586 registros de patentes. Estas tendencias reflejan la importancia que está teniendo esta nueva área en el sector de la investigación y el desarrollo tecnológico.

\section{Principales países e instituciones con desarrollo tecnológico}

De los países u oficinas con mayor número de patentes registradas en el área de estudio,
Estados Unidos y la oficina Tratado de Cooperación en materia de Patentes, en sus siglas en inglés PCT, lideran las cifras: el primero con 2.205 y el segundo con 1.178 invenciones protegidas, respectivamente. Por otro lado, Australia, la Oficina Europea de Patentes y Canadá, se ubican en el tercer, cuarto y quinto lugar reportando cifras de 293, 288 y 152 invenciones, respectivamente. Los demás países e instituciones se muestran en el Gráfico 6.

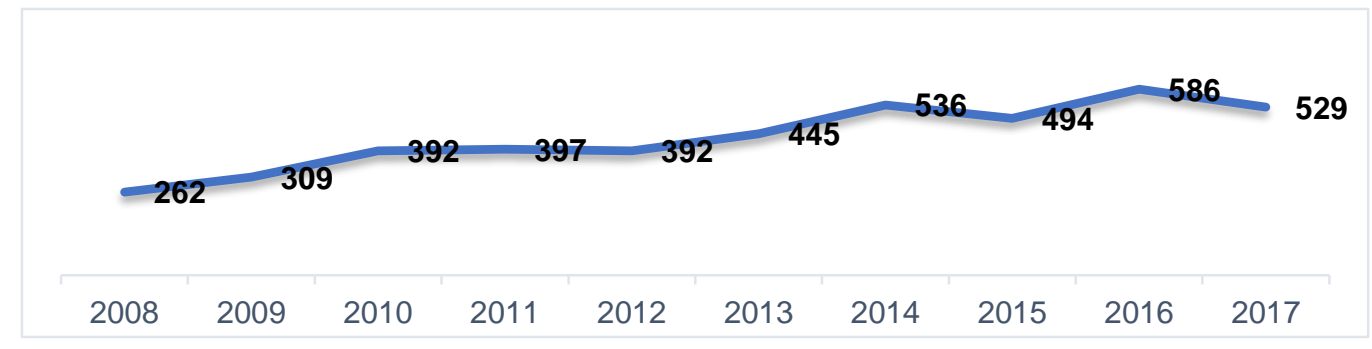

Gráfico 5. Comportamiento de las patentes publicadas a través de los años

Graph 5. Behavior of patents published over the years

Fuente: elaboración propia con base a WIPO - Patenstcope ${ }^{\circledR}$

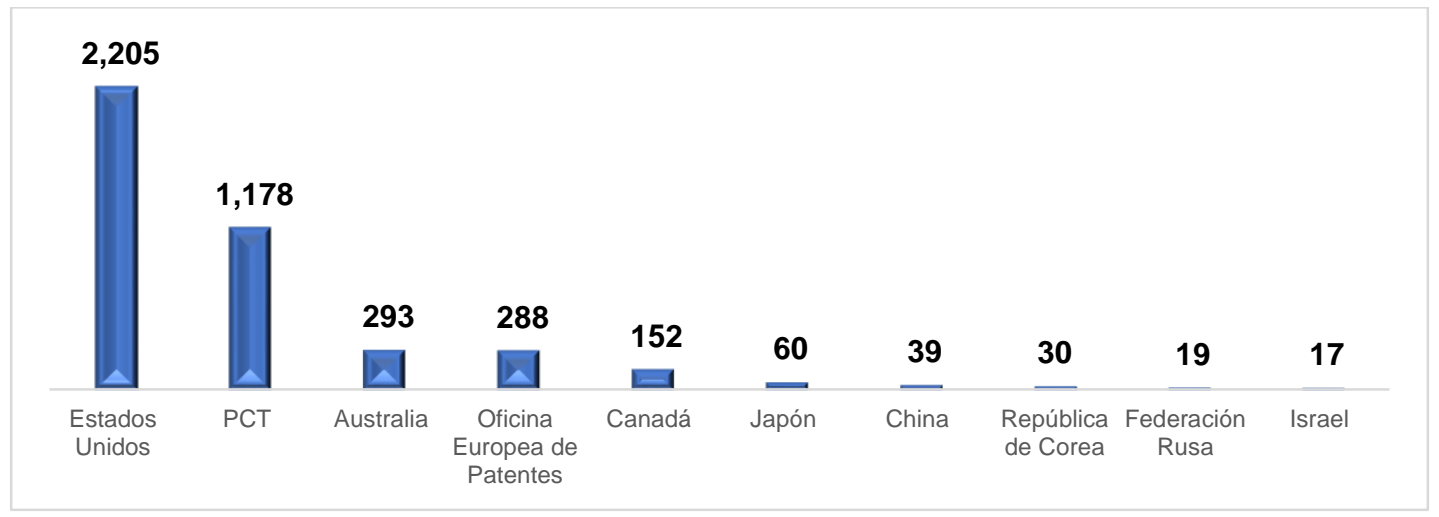

Gráfico 6. Países e instituciones líderes con desarrollo tecnológico en el área

Graph 6. Leading countries and institutions with technological development in the field Fuente: elaboración propia con base a WIPO - Patenstcope

Red de las principales tecnologías identificadas (Potenciales oportunidades de innovación)

En esta red se muestran las principales tecnologías identificadas en el estudio según los resultados de la producción científica y tecnológica. Para el diseño de esta red, se basó de la información obtenida de las ecuaciones de búsqueda y someterlas a una herramienta de creación y visualización de grafos denominada VOSviwer". Como se puede observar en la Figura 5, alrededor de nanotecnología en la industria de alimentos se han empezado a desarrollar: nanopartículas, nanomateriales,

nanocompuestos, 
nanoemulsión, nanoencapsulación, innovación para la industria alimenticia y otras microencapsulación, nanorecubrimientos y áreas como la agricultura, la ingeniería, la nanosensores. Estas tecnologías están en biología y farmacología, la física, química entre constante fase de investigación y desarrollo y otras. se convierten en potenciales oportunidades de

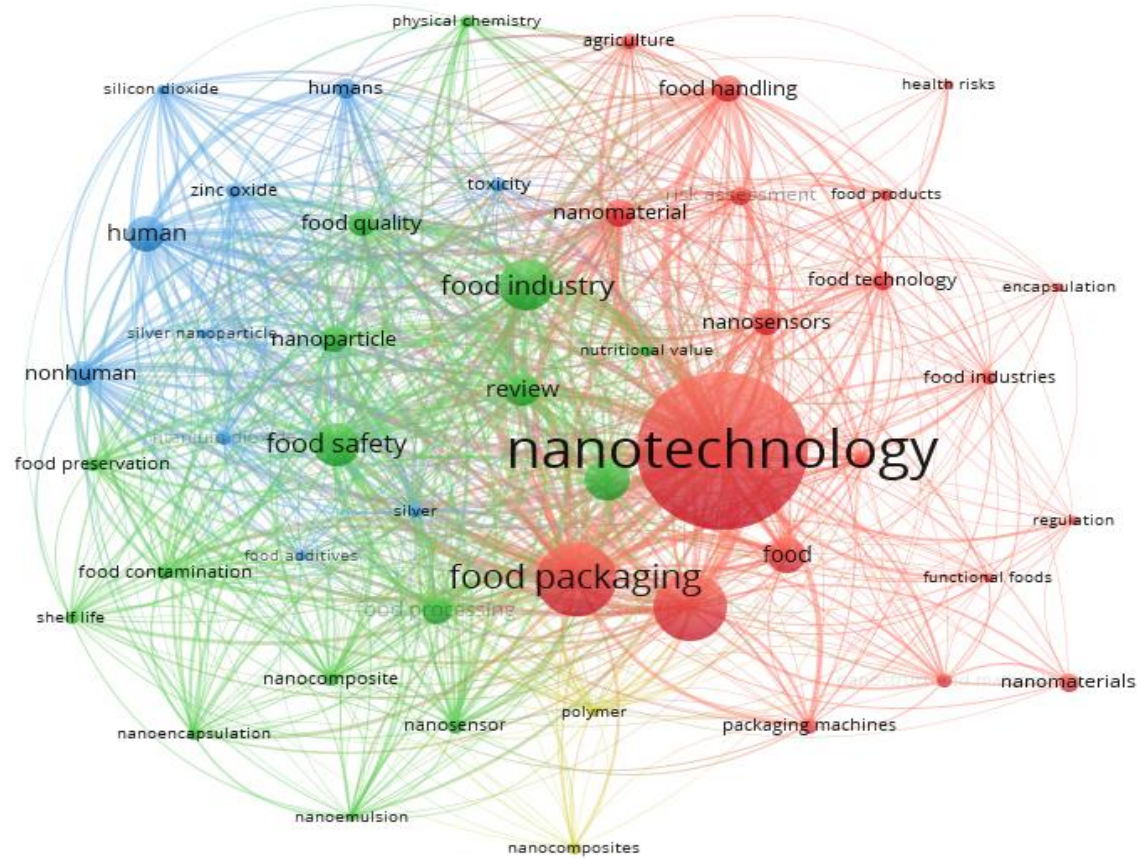

Figura 5. Red de las principales tecnologías identificadas en las bases de datos

Figure 5. Network of main technologies identified in the databases Fuente: elaboración propia.

\section{CONCLUSIONES Y DISCUSIONES}

Como se ha podido conocer en los resultados de este estudio, el uso de la nanotecnología en la industria alimentaria está emergiendo rápidamente como una nueva área de investigación y desarrollo para obtener y/o fabricar alimentos más saludables, duraderos y para mejorar su inocuidad y procesos de trazabilidad (Drew \& Hagen, 2016; Lauterwasser, 2016).

Lo anterior se relacionan con las investigaciones de Kalpana Sastry, Anshul, \& Rao (2013); Naranjo et al. (2014); Colica et al. (2018); Changan Li, Hai Chen, Bin Chen \& Guanghua Zhao (2018) Cummings, Chuah, \& Ho (2018) Hamad et al. (2018), donde resaltan que varios grupos de investigación, incluidas empresas privadas de la industria, han iniciado programas de investigación y desarrollo para explorar el amplio alcance de la nanotecnología en la cadena de valor del procesamiento y la fabricación de alimentos.

Las tendencias en producción científica y tecnológica muestran que la nanotecnología se configura como una importante caja de herramientas emergente $y$ en rápido desarrollo, que tiene aplicaciones novedosas y únicas para la ciencia de los alimentos, como se señala en los estudios de Sadeghi et al., (2017), quienes sostienen que los rápidos e impresionantes avances en esta área han dado lugar a nuevos prototipos experimentales de tecnologías y productos. 
Por otro lado, con respecto a los resultados del estudio, se pudo conocer que los países con mayor producción y desarrollo científico, generalmente no son los de mayor desarrollo tecnológico, como es el caso de India y Bélgica, por mencionar algunos. Estos países figuran en el top 10 como los líderes en generación de conocimiento en la industria, sin embargo, este indicador no guarda relación con el desarrollo tecnológico.

Según las tendencias analizadas, son especialmente los países industrializados los que han destinado mayor esfuerzo en el fomento de actividades de I+D en nanotecnología, y son estos quienes poseen el mayor número de patentes concedidas, concordando con los resultados de Naranjo et al. (2014), en los que se evidenciaron grandes diferencias en materia de desarrollo científico y tecnológico entre los países industrializados y los caracterizados por su rezago tecnológico con base al tema de análisis.

En este orden, el mayor desarrollo científicotecnológico en este campo de estudio estuvieron relacionados con el diseño de nuevos nanomateriales y partículas, así como la creación de nuevas nanosustancias y nuevos sistemas de envasado para mejorar la conservación e inocuidad de los alimentos. Estos desarrollos se relacionan con lo encontrado en los estudios de Molins (2008); Amini, Seyed Mohammad; Gilaki, Marzieh; Karchani (2014); Shankar \& Rhim, (2016); Peter Viberg (2017); Andrew B., Cundy (2017); Dilmaçünal, T. (2017); Kuswandi, Futra, \& Heng, (2017); Cerqueira et al., 2017; Hamad, Han, Kim, \& Rather (2018); Thiruvengadam, \& Chung (2018), en donde el uso de la nanotecnología en la industria de alimentos ha tenido diversas aplicaciones, como para diseñar nuevos ingredientes alimentarios a través de nanocompuestos, nanopartículas y nanomateriales, implementer mejoras en los sistemas de suministro de nutrientes $y$ bioactivos, en la encapsulación y microencapsulación de textura y sabor, en el control microbiológico, en el procesamiento y envasado de alimentos y en biosensores específicos altamente sensibles que pueden usarse para detectar patógenos, alérgenos, contaminantes y degradantes que pueden afectar la calidad y la seguridad de los alimentos.

Las aplicaciones de la nanotecnología en los alimentos han aumentado rápidamente en las últimas décadas y su constante evolución ha tenido una gran contribución en el procesamiento de alimentos, el paquete y la conservación de los mismos. Esta evolución en la industria alimentaria ha contribuido eficientemente en el procesamiento, empaquetado y conservación de alimentos. Estos recientes avances sugieren aplicaciones nuevas e innovadoras y se configura como un método eficiente no sólo para la industria alimentaria sino también para otros campos, como como la agricultura, la ingeniería, la biología y farmacología, la física, química entre otras.

Sin embargo, a pesar del gran avance $y$ desarrollo tecnológico de la nanotecnología en la industria de alimentos, algunos estudios señalan los posibles impactos y riesgos que puede afectar la seguridad alimentaria (Noormans, 2010; Veiga-Álvarez et al., 2015; Drew \& Hagen, 2016; Rolando, 2016).

Con respecto a su impacto, algunos autores plantean que esta nueva tecnología cambiaría la forma del manejo, producción, trazabilidad e inocuidad de los alimentos así como la modificación a nivel molecular de los mismo (Bouwmeester et al., 2007; Noormans, 2010; Veiga-Álvarez et al., 2015); a su vez modificaría nuestra dieta alimentaria bebido al desarrollo de nuevos suplementos alimenticios, nanosustancias y nanomateriales lo que puede crear riesgos a la adicción y a la salud durante y después de la manipulación de algunos de sus compuesto, nano-partículas y sustancias (Smith \& Raven, 2012; Drew \& Hagen, 2016; Lauterwasser, 2016). 
Los estudios de (Bouwmeester et al., 2007; Drew \& Hagen, 2016; Lauterwasser, 2016) señalan que la nanotecnología en la industria de alimentos, al igual que la mayoría de las tecnologías nuevas y en evolución y de los beneficios que pueda tener, se ha estudiado poco sobre las consecuencias y riesgos derivados en su aplicación, desarrollo y modificación de alimentos. Por lo tanto, el foco del debate se centra en generar acciones inmediatas por parte de los creadores de políticas y científicos, así como crear nuevos marcos regulatorios y legislativos sólidos donde se incorporen Buenas Prácticas de Laboratorio (BPL) y procedimientos estándar dentro de este nuevo desarrollo con el fin de minimizar y controlar los riesgos asociados a esta nueva tecnología en la industria.

Finalmente, en el estudio se pudo identificar ocho potenciales oportunidades de innovación en el área: nanopartículas, nanomateriales, nanocompuestos, nanoemulsión, nanoencapsulación, microencapsulación, nanorecubrimientos y nanosensores, las cuales están influyendo notablemente en el desarrollo de la industria de alimentos y se han convertido en elementos claves de investigación y desarrollo tecnológico a nivel mundial.

\section{REFERENCIAS}

Andrew, B. (2017). Small Solutions to Large Problems? Nanomaterials and Nanocomposites in Effluent, Water, and Land Management. https://doi.org/10.1016/B978-0-32342980-1.00003-0

Aouada, de M. (2015). Nanotechnology Applied in Agriculture: Controlled Release of Agrochemicals. In: Rai M., Ribeiro C., Mattoso L., Duran N. (eds) Nanotechnologies in Food and Agriculture. Springer, Cham. https://doi- org.itm.elogim.com:2443/10.1007/978-

3-319-14024-7_5

Amini, Seyed Mohammad; Gilaki, Marzieh; Karchani, M. (2014). Safety of nanotechnology in food industries. Electronic Physician, 6(4), 962-968. https://doi.org/10.14661/2014.962-968

Barbosa, A. F. (2012). Vigilancia tecnológica: metodologías y aplicaciones.

Bautista, L., Molina, L., Niembro, S., García, J. M., López, J., \& Vílchez, A. (2017). Coatings and Inks for Food Packaging Including Nanomaterials. Emerging Nanotechnologies in Food Science. Elsevier Inc. https://doi.org/10.1016/B978-0-32342980-1.00008-X

Bowman, D. M., \& Ludlow, K. (2017). Ensuring Food Safety: General Principles for Safeguarding What You Eat Including the Role of Food Labels. Emerging Nanotechnologies in Food Science. Elsevier Inc. https://doi.org/10.1016/B978-0-32342980-1.00009-1

Bouwmeester, H., Dekkers, S., Noordam, M., Hagens, W., Bulder, A., Heer, C. De, ... Sips, A. (2007). Health impact of nanotechnologies in food production, 191. Recuperado de www.rivm.nl

Busquets, R. (2017). Analysis of Nanomaterials in Food. Emerging Nanotechnologies in Food Science. Elsevier Inc. https://doi.org/10.1016/B978-0-32342980-1.00004-2

Busquets, R., \& Mbundi, L. (2017). Concepts of Nanotechnology. Emerging Nanotechnologies in Food Science. Elsevier Inc. https://doi.org/10.1016/B978-0-32342980-1.00001-7 
Cerqueira, M. Â., Pinheiro, A. C., Ramos, O. L., Silva, H., Bourbon, A. I., \& Vicente, A. A. (2017). Advances in Food Nanotechnology. Emerging Nanotechnologies in Food Science. Elsevier Inc. https://doi.org/10.1016/B978-0-32342980-1.00002-9

Changan Li, Hai Chen, Bin Chen \& Guanghua Zhao (2017). Highly fluorescent gold nanoclusters stabilized by food proteins: From preparation to application in detection of food contaminants and bioactive nutrients. Critical Reviews in Food Science and Nutrition, 58(5), 689699.

https://doi.org/10.1080/10408398.2016. 1213698

Colica, C., Aiello, V., Boccuto, L., Vecchio, I., Abenavoli, L. (2018). The role of Nanotechnology in food safety. Minerva Biotecnologica 30(2), 69-73.

Cundy, A. B. (2017). Small Solutions to Large Problems? Nanomaterials and Nanocomposites in Effluent, Water, and Land Management. Emerging Nanotechnologies in Food Science. Elsevier Inc. https://doi.org/10.1016/B978-0-32342980-1.00003-0

Cummings, C. L., Chuah, A. S. F., \& Ho, S. S. (2018). Protection Motivation and Communication through Nanofood Labels: Improving Predictive Capabilities of Attitudes and Purchase Intentions toward Nanofoods. Science Technology and Human Values, 1-29. https://doi.org/10.1177/0162243917753 991

Dilmaçünal, T. (2017). Intelligent Systems in the Food Packaging Industry: Contaminant Sensors and Security/Anticounterfeiting
Devices. https://doi.org/10.1016/B978-012-811942-6.00014-5

Drew, R., \& Hagen, T. (2016). Potential Health Risks Associated with Nanotechnologies in Existing Food Additives.

Echegoyen, Y. (2015). Nano-developments for Food Packaging and Labeling Applications. In: Rai M., Ribeiro C., Mattoso L., Duran N. (eds). Nanotechnologies in Food and Agriculture. Springer, Cham. https://doiorg.itm.elogim.com:2443/10.1007/9783-319-14024-7_7

Eduok, S., \& Coulon, F. (2017). Microbiological Toxicity of Nanoparticles. Emerging Nanotechnologies in Food Science. Elsevier Inc. https://doi.org/10.1016/B978-0-32342980-1.00006-6

Elango, B., Rajendran, P., \& Bornmann, L. (2015). A scientometric analysis of international collaboration and growth of literature at the macro level. Malaysian Journal of Library and Information Science, 20(2), 41-50.

Eleftheriadou, M., Pyrgiotakis, G., \& Demokritou, P. (2017). Nanotechnology to the rescue: using nano-enabled approaches in microbiological food safety and quality. Current Opinion in Biotechnology, 44, 87-93. https://doi.org/10.1016/j.copbio.2016.11 .012

Elsevier. (2015). Funcionalidades avanzadas en Scopus, 1-58.

Erdem, S. (2015). Consumers' Preferences for Nanotechnology in Food Packaging: A Discrete Choice Experiment. Journal of Agricultural Economics, 66(2), 259-279. https://doi.org/10.1111/14779552.12088 
Foladori, G., \& Invernizzi, N. (2012). Implicaciones sociales y ambientales del desarrollo de las nanotecnologías en América Latina y el Caribe. México y Curitiba, Brasil: ReLANS e, IPEN. Recuperado de om http://www6.reluita.org/nanotecnologia/Nanotecnologia2.pdf

Fornari, E., Fornari, D., Grandi, S., Menegatti, M., \& Hofacker, C. F. (2016). Article information. https://doi.org/10.1108/IJRDM-07-20150103

Fundación Vasca para la Seguridad Alimentaria. (2012). La aplicación de las nuevas tecnologías en la industria alimentaria. Recuperado de http://www.elika.eus/datos/articulos/Arc hivo993/Artículo_nanotecnología alimentaria.pdf

Gomes, R. C., Pastore, V. A. A., Martins, O. A., \& Francisco, G. (2015). Aplicações da nanotecnologia na indústria de alimentos. Uma Revisão, 8, 1-8.

Granqvist, N., \& Ritvala, T. (2016). Beyond Prototypes: Drivers of Market Categorization in Functional Foods and Nanotechnology. Journal of Management Studies, 53(2), 210-237. https://doi.org/10.1111/joms.12164

Hamad, A. F., Han, J. H., Kim, B. C., \& Rather, I. A. (2018). The intertwine of nanotechnology with the food industry. Saudi Journal of Biological Sciences, 25(1), 27-30.

https://doi.org/10.1016/j.sjbs.2017.09.004

He, X., \& Hwang, H. M. (2016). Nanotechnology in food science: Functionality, applicability, and safety assessment. Journal of Food and Drug Analysis, 24(4), 671-681.

https://doi.org/10.1016/j.jfda.2016.06.00
1

Jurewicz, M. (2017). Uregulowania prawne wykorzystania nanotechnologii w produkcji materiałów i wyrobów z tworzyw polimerowych przeznaczonych do kontaktu z żywnością, (2), 144-148.

Kalpana Sastry, R., Anshul, S., \& Rao, N. H. (2013). Nanotechnology in food processing sector-An assessment of emerging trends. Journal of Food Science and Technology, 50(5), 831-841. https://doi.org/10.1007/s13197-0120873-y

Kuswandi, B., Futra, D., \& Heng, L. Y. (2017). Nanosensors for the Detection of Food Contaminants. Nanotechnology Applications in Food: Flavor, Stability, Nutrition and Safety. Elsevier Inc. https://doi.org/10.1016/B978-0-12811942-6.00015-7

Lacey, J. (2017). Bioavailability of Nanomaterials and Interaction With Cells. Emerging Nanotechnologies in Food Science. Elsevier Inc. https://doi.org/10.1016/B978-0-32342980-1.00005-4

Lauterwasser, C. (2016). Small sizes that matter: Opportunities and risks of Nanotechnologies. Allianz, 46.

Macías. (2001). Papel de la informetría y de la cienciometría y su perspectiva. Scielo, 3541.

Masami et al. (2013). Nanotechnology and Public Health: Contributions, Promises, and Premises. https://doi.org/10.1002/9781118451915 .ch3

Molina, J. L. (2004). La ciencia de las redes. Apuntes de Ciencia y Tecnologia, (11), 3642. 
Molins, R. (2008). Oportunidades y amenazas de la nanotecnología. Comuniic4, 38-53. Retrieved from ricardo.molins@iica.int

Noormans, A. G. (2010). Impact of Nanotechnology in Food Production Impacto de la Nanotecnología en la Producción de Alimentos. Lámpsakos, (4), 2145-4086.

Naranjo, M., Cecilia, K., Palacios, C., Lorena, M., Correa, B., Mesa, R., Palacios, C. (2014). Tendencias investigativas de la nanotecnología en empaques y envases para alimentos.

Otles S., Yalcın, B. (2015). Papel estratégico del nanobiosensor en los alimentos: beneficios y cuellos de botella. En: Rai M., Ribeiro C., Mattoso L., Duran N. (eds). Nanotecnologías en alimentación y agricultura. Springer, Cham. https://doiorg.itm.elogim.com:2443/10.1007/978-

3-319-14024-7_8

Pathak (2017). Nanoemulsions and Their Stability for Enhancing Functional Properties of Food Ingredients. Nanotechnology Applications in Food. Flavor, Stability, Nutrition and Safety. https://doi.org/10.1016/B978-0-12811942-6.00005-4

Peter Viberg. (2017). Emerging Nanotechnologies in Food Science. https://doi.org/10.1016/B978-0-32342980-1.00010-8

Pinto, A. L., \& González-Aguilar, A. (2014). Visibilidad de los estudios en análisis de redes sociales en América del Sur: Su evolución y métricas de 1990-2013. Transinformação, Campinas, 26(3), 253267.

Robledo, S., Osorio, G. A., \& López, C. (2014). Networking en pequeña empresa: una revisión bibliográfica utilizando la teoria de grafos. Revista Vínculos, 11(2), 6-16. https://doi.org/https://doi.org/10.14483/ issn.2322-939X

Robles-García, M. A., Rodríguez-Félix, F., Márquez-Ríos, E., Aguilar, J. A., BarreraRodríguez, A., Aguilar, J., ... Del-ToroSánchez, C. L. (2016). Applications of Nanotechnology in the Agriculture, Food, and Pharmaceuticals. Journal of Nanoscience and Nanotechnology, 16(8), 8188-8207.

https://doi.org/10.1166/jnn.2016.12925

Rolando, C. (2016). Nanotecnología en la industria alimentaria. Universidad Politécnica de Madrid, 1-32.

Rodríguez, J. (2015). A critical review of SClmago Journal \& Country Rank. Research Evaluation, 24(4), 343-354. https://doi.org/10.1093/reseval/rvu008

Sadeghi, R., Rodríguez, R. J., Yao, Y., \& Kokini, J. L. (2017). Advances in Nanotechnology as they Pertain to Food and Agriculture: Benefits and Risks, (January), 1-26. https://doi.org/10.1146/annurev-food041715-033338

Serena, P. A. (2002). Nanociencia y nanotecnología: aspectos generales. Encuentros Multidisciplinares, 1-12. https://doi.org/https://dialnet.unirioja.es /servlet/articulo?codigo=285405

Shankar, S., \& Rhim, J.-W. (2016). Polymer Nanocomposites for Food Packaging Applications. Functional and Physical Properties of Polymer Nanocomposites. Elsevier Inc. https://doi.org/10.1002/9781118542316 .ch3

Singh, H. (2016). Review Article Nanotechnology Applications in Functional Foods; Opportunities and 
Challenges, 21(February), 1-8. https://doi.org/10.3746/pnf.2016.21.1.1

Singh, T., Shukla, S., Kumar, P., Wahla, V., \& Bajpai, V. K. (2017). Application of nanotechnology in food science: Perception and overview. Frontiers in Microbiology, 8(AUG), 1-7. https://doi.org/10.3389/fmicb.2017.01501

Smith, A., \& Raven, R. (2012). What is protective space? Reconsidering niches in transitions to sustainability. Research Policy, 41(6), 1025-1036. https://doi.org/10.1016/j.respol.2011.12. 012

Sodano, V., Gorgitano, M. T., Quaglietta, M., \& Verneau, F. (2016). Regulating food nanotechnologies in the European Union: Open issues and political challenges. Trends in Food Science and Technology, 54 , 216-226. https://doi.org/10.1016/j.tifs.2016.05.022

Sougata., Gandhi \& Jana (2017). Nanotechnology in Bioactive Food Ingredients: Its Pharmaceutical and Biomedical Approaches. Nanotechnology Applications in Food. Flavor, Stability, Nutrition and Safety. Pages 21-41. https://doi.org/10.1016/B978-0-12811942-6.00002-9

Steenis, N., \& Fischer, A. (2016). Consumer attitudes towards nanotechnology in food products: an attribute-based analysis. British Food Journal, 118, 1254-1267. https://doi.org/10.1108/BFJ-09-20150330
Thiruvengadam, M., Rajakumar, G., \& Chung, I.M. (2018). Nanotechnology: current uses and future applications in the food industry. 3 Biotech, 8(1), 74. https://doi.org/10.1007/s13205-0181104-7

Tomiko (2013). The Challenge of Nanotechnology-Derived Food: Addressing the Concerns of the Public. https://doi.org/10.1002/9781118451915 .ch2

Wasserman, S., \& Faust, K. (2011). Social network analysis: Methods and applications. Cambridge University Press, 1 ,

116. https://doi.org/10.1525/ae.1997.24.1.219

Veiga-Álvarez, Á., Sánchez-de-Alcázar, D., Martínez-Negro, M., Barbu, A., GonzálezDíaz, J. B., \& Maquea-Blasco, J. (2015). Riesgos para la salud y recomendaciones en el manejo de nanopartículas en entornos laborales. Medicina y Seguridad Del Trabajo, 61(239), 143-161. https://doi.org/10.4321/S0465546X2015000200002

WIPO. (2017). Guía del usuario. Recuperado de:

http://www.wipo.int/hague/es/guide/

Yandy, O., \& Goyeneche, R. (2015). Instituto de Estudios Ambientales (IDEA), Facultad de Ciencias Económicas Universidad Nacional de Colombia. Bogotá, Colombia. 